\title{
LETTER
}

Multiple myeloma, gammopathies

\section{Outcomes among newly diagnosed AL amyloidosis patients with a very high NT-proBNP: implications for trial design}

\author{
I. Vaxman $\mathbb{D}^{1,2,3} \cdot$ S. K. Kumar $\mathbb{D}^{1} \cdot$ F. Buadi $\mathbb{D}^{1} \cdot$ M. Q. Lacy ${ }^{1} \cdot$ D. Dingli $\mathbb{D}^{1} \cdot$ Y. Hwa ${ }^{1} \cdot$ A. Fonder ${ }^{1} \cdot$ M. Hobbs ${ }^{1} \cdot$ \\ S. Hayman ${ }^{1} \cdot$ T. Kourelis $\mathbb{D}^{1} \cdot$ R. Warsame ${ }^{1} \cdot$ E. Muchtar $\mathbb{D}^{1} \cdot$ N. Leung $\mathbb{D}^{1} \cdot$ P. Kapoor ${ }^{1} \cdot$ M. Grogan ${ }^{4} \cdot$ R. Go $\mathbb{D}^{1} \cdot$ \\ Y. Lin $^{1} \cdot$ W. Gonsalves ${ }^{1} \cdot$ M. Siddiqui $\mathbb{D}^{1} \cdot$ R. A. Kyle ${ }^{1}$ - S. V. Rajkumar ${ }^{1} \cdot$ M. A. Gertz $\mathbb{D}^{1} \cdot$ A. Dispenzieri ${ }^{1}$
}

Received: 5 February 2021 / Revised: 30 April 2021 / Accepted: 10 May 2021 / Published online: 21 May 2021

(c) The Author(s) 2021. This article is published with open access

\section{To the Editor:}

Prognosis in AL amyloidosis is variable and is a function of plasma cell biology (serum-free light chains, genetics, bone marrow plasma cell burden, depth of hematologic response to treatment and organ involvement) [1]. There are two commonly used prognostic scores for staging in AL amyloidosis [2-4], both use cardiac biomarkers to predict survival: European modification of Mayo 2004 and Mayo 2012, which uses slightly different cut-offs and adds the difference between involved and uninvolved light chain (dFLC) into the model.

It is well documented that overall survival (OS) in AL amyloidosis is improving over time, in part due to earlier diagnosis and in part due to more effective therapies [5, 6]. For the purposes of clinical trials, patients with the highestrisk disease are often excluded from trial participation [7, 8]. An N-terminal pro-brain natriuretic peptide (NTproBNP) of $8500 \mathrm{ng} / \mathrm{L}$ or higher is the criterion most often used for exclusion from clinical trials due to high rates of early death [4, 8]. In an effort to set the stage for better clinical trial design, we aimed to characterize the outcomes of patients with very high (VH) NT-proBNP ( $\geq 8500 \mathrm{ng} / \mathrm{L})$

Supplementary information The online version contains supplementary material available at https://doi.org/10.1038/s41375021-01297-z.

A. Dispenzieri

dispenzieri.angela@mayo.edu

1 Division of Hematology, Mayo Clinic, Rochester, MN, USA

2 Institute of Hematology, Davidoff Cancer Center, Rabin Medical Center, Petah Tikva, Israel

3 Sackler Faculty of Medicine, Tel-Aviv University, Tel-Aviv, Israel

4 Department of Cardiovascular Medicine, Mayo Clinic, Rochester, MN, USA and evaluate the prognostic scores in terms of their ability to stage discriminate patients with and without VH NTproBNP.

We retrospectively identified newly diagnosed $\mathrm{AL}$ amyloidosis patients who were diagnosed between January 2012 and July 2020 ( $n=1290)$. Patients were excluded if they were not seen at Mayo Clinic within 90 days of diagnosis $(n=291)$ and if they did not have baseline biomarkers $(n=170)$, leaving 829 patients for our analysis.

The diagnosis and staging of AL amyloidosis were according to consensus criteria [2-4, 9]. Thresholds for troponins and BNPs were corrected using a conversion method previously described by our group [10]. The vast majority of patients had troponin T measured, and for them, the $0.025 \mathrm{mcg} / \mathrm{L}$ and the $0.035 \mathrm{mcg} / \mathrm{L}$ cut-points were used for the 2012 and 2004 staging systems, respectively. A minority of patients did not have troponin $\mathrm{T}$, but rather high sensitivity troponin $\mathrm{T}(n=129)$ or troponin $\mathrm{I}(n=23)$. For patients with high sensitivity troponin $\mathrm{T}$, cut-points of 40 and $50 \mathrm{ng} / \mathrm{L}$ were used, respectively, for the 2012 and 2004 systems, and for patients with troponin I only, a cutpoint of $0.1 \mathrm{mcg} / \mathrm{L}$ was used for both systems. In the three patients with no NT-proBNP but with BNP, 400 and $81 \mathrm{ng} /$ L were used respectively for the 2012 and 2004 systems; otherwise, the $1800 \mathrm{ng} / \mathrm{L}$ and $332 \mathrm{ng} / \mathrm{L}$ cut-offs were used for NT-proBNP. A BNP of $>700 \mathrm{ng} / \mathrm{L}$ was considered equivalent to NT-proBNP $\geq 8500 \mathrm{ng} / \mathrm{L}$. VH NT-proBNP was defined as NT-proBNP $\geq 8500 \mathrm{ng} / \mathrm{L}$ or equivalent.

First-line treatments were chosen by treating physicians based on the extent of cardiac involvement, age, performance status, drug availability and patient's preference. Organ involvement was defined according to existing criteria [9]. Patients underwent ASCT if they were eligible according to the mSMART criteria. [1]

Patient and disease factors were compared for categorical and continuous variables using $\left(\chi^{2}\right.$, or Fisher's exact) and ( $t$ test, or Wilcoxon signed-rank test), respectively. OS was 
A.
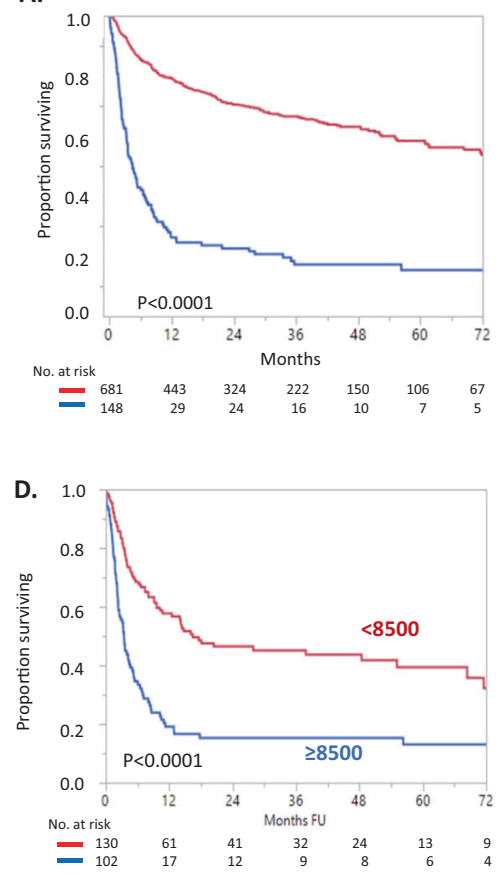

B.

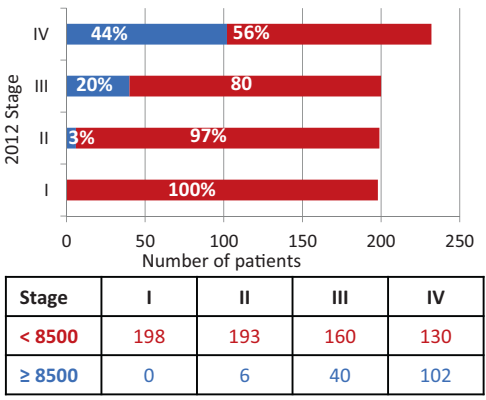

E.

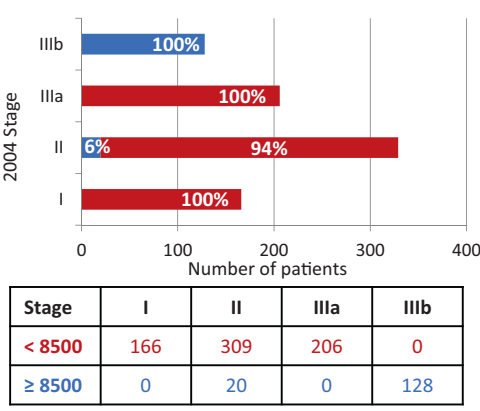

C.

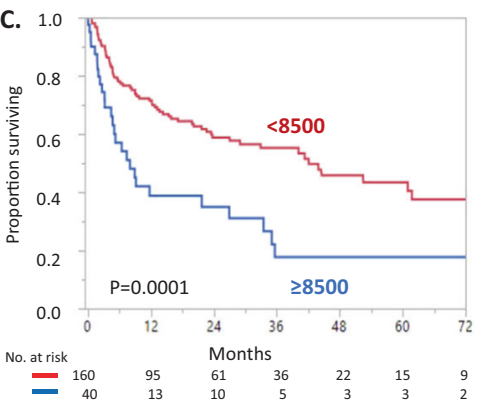

F.

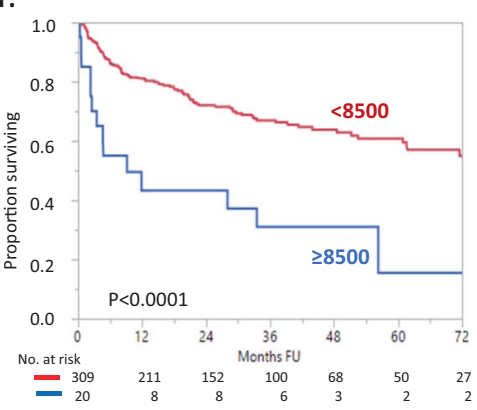

Fig. 1 Interaction between biomarker stage and very high levels of NT-proBNP. A Survival parsed by NT proBNP $\geq 8500 \mathrm{pg} / \mathrm{mL}$. B Proportion of patients surviving by Mayo 2012 stage parsed by NTproBNP. C Overall survival of the Mayo 2012 Stage III with NTproBNP parsed by NT-proBNP. D Overall survival of the Mayo 2012
Stage IV with NT-proBNP parsed by NT-proBNP. E Proportion of patients surviving by European modification of Mayo 2004 staging and parsed by NT-proBNP. F Overall survival of the Mayo 2004 Stage II patients parsed by NT-proBNP.

Table 1 Early mortality based on NT-proBNP and stage.

\begin{tabular}{|c|c|c|c|c|c|c|c|}
\hline & & & \multirow[t]{2}{*}{$N$} & \multicolumn{4}{|l|}{$\%$ Dead at } \\
\hline & & & & 3 months & 6 months & 9 months & 12 months \\
\hline \multirow[t]{9}{*}{ NT-proBNP $\geq 8500$} & All patients & & 148 & 38 & 58 & 68 & 74 \\
\hline & 2012 Stage & I & 0 & - & - & - & - \\
\hline & & II & 6 & 17 & 17 & 28 & 38 \\
\hline & & III & 40 & 27 & 45 & 56 & 61 \\
\hline & & IV & 102 & 45 & 66 & 76 & 82 \\
\hline & 2004 Stage & I & 0 & - & - & - & - \\
\hline & & II & 20 & 32 & 45 & 50 & 57 \\
\hline & & IIIa & 0 & - & - & - & - \\
\hline & & IIIb & 128 & 39 & 60 & 72 & 77 \\
\hline \multirow[t]{9}{*}{ NT- proBNP $<8500$} & All patients & & 681 & 7 & 15 & 18 & 21 \\
\hline & 2012 Stage & I & 198 & 2 & 4 & 5 & 7 \\
\hline & & II & 193 & 4 & 8 & 13 & 14 \\
\hline & & III & 160 & 10 & 22 & 26 & 28 \\
\hline & & IV & 130 & 17 & 32 & 38 & 43 \\
\hline & 2004 Stage & I & 166 & 1 & 2 & 3 & 4 \\
\hline & & II & 309 & 6 & 14 & 18 & 19 \\
\hline & & IIIa & 206 & 13 & 26 & 33 & 37 \\
\hline & & IIIb & 0 & - & - & - & - \\
\hline
\end{tabular}

$N$ number, $N T$-proBNP N-terminal pro-brain natriuretic peptide. 
defined as the time from diagnosis to death from any cause. Kaplan-Meier method was used for OS analysis and differences in survival were determined by Log-Rank. All statistical tests were two-sided and $P$ values of $<0.05$ were considered to be significant. Statistical analysis was carried out using JMP 14 (SAS Institute, Cary, NC) statistical software.

Of the 829 patients, $148(18 \%)$ had a VH NT-proBNP $(\geq 8500 \mathrm{ng} / \mathrm{L})$. Patients with VH NT-proBNP were older (67 versus 64 years, $P=0.004)$. Supplementary Table 1 shows the characteristics of the two patient subgroups. The $\mathrm{VH}$ NT-proBNP subgroup had higher baseline median levels of bilirubin $(P<0.001)$, alkaline phosphatase $(P<0.001)$ and dFLC $(P<0.001)$. For patients without VH NT-proBNP, the median urinary protein was significantly higher $(P=$ $0.006)$, the serum creatinine was lower $(P<0.0001)$ and glomerular filtration rate (GFR) was higher $(P<0.001)$. Patients with VH NT-proBNP were older than patients with NT-proBNP $<8500 \mathrm{ng} / \mathrm{L}$. This may reflect delayed diagnosis in older patients, seeing that symptoms in amyloidosis are non-specific and are often attributed to comorbidities [11], which are more common in an elderly population.

With a median follow-up of 30 months, median OS for VH NT-proBNP patients was 4.4 months in contrast to patients without VH NT-proBNP at 77.3 months $(P<$ 0.0001) (Fig. 1A). Breakdown of early death for patients with VH NT-proBNP by stage is shown in Table 1 . Seventy-four percent of the patients with VH NT-proBNP died in the first year. Of Mayo 2012 patients staged I, II, III, and IV, the percent of patients with VH NT-proBNP was $0 \%, 3 \%, 20 \%$, and $44 \%$ (Fig. 1B). Patients with VH-NTproBNP had early death rates comparable to (or even worse than) patients without VH NT-proBNP but who were a stage higher. For example, 3-month mortality for Mayo 2012 Stage III and VH NT-proBNP was 27\% as compared to $17 \%$ among Stage IV without VH-NT-proBNP (Table 1; Fig. 1C, D). A similar pattern was seen for the European modification of the Mayo 2004 system though the addition of the VH NT-proBNP qualifier to the European modification of the Mayo 2004 staging system had less overall impact since it is already part of the definition (Table 1; Fig. 1E, F).

Thirty-nine patients (26\%) in the VH NT-proBNP cohort lived greater than 1 year. Patients that survived 1 year or longer had lower levels of bilirubin $(P=0.005)$, uric acid $(P=0.0024)$, GFR levels $(P=0.04)$, and lower albumin $(P$ $=0.02)$, but higher urinary protein $(P=0.02)$. These findings suggest that the VH NT-proBNP patients had delayed diagnosis and more extensive disease at diagnosis and that patients with renal presentations may have earlier diagnosis. Also of note, among the VH-NT-proBNP group there was no difference in OS between patients with low GFR and those with normal kidney function.
In terms of treatment, there were missing data about firstline therapy for 246 patients, 207 (31\%) in the $<8500$ group and $39(26 \%)$ in the VH-NT-proBNP group. Only $3 \%$ of the VH NT-proBNP patients received an ASCT in contrast to $29 \%$ of patients without VH NT-proBNP $(P<0.0001)$. The majority of patients received alkylators and bortezomib as the first-line treatment. Among the VH NT-pro BNP group, 76 patients received the combination of PI and an alkylator, 14 received PI only and 14 received alkylators only. Among patients that received therapy, there was no difference in bortezomib use between the 1-year survivors and nonsurvivors $(81 \%$ versus $78 \%, P=0.74)$. None of the five patients who received daratumumab-based therapy at the first line had a VH NT-proBNP. Forty patients, only 3 of whom had VH NT-proBNP, received daratumumab as part of second-line therapy.

Prospective data about daratumumab in patients with $\mathrm{VH}$ NT-proBNP are lacking, as they were excluded from the ANDROMEDA trial [7], The best data for daratumumab use in AL amyloidosis patients with VH NT-proBNP is a retrospective study from Heidelberg, which included 168 patients with relapsed disease treated with daratumumab and dexamethasone with (DVD) or without (DD) bortezomib [12]; 25 patients had NT-proBNP $>8500 \mathrm{ng} / \mathrm{L}$. For patients with VH NT-proBNP, the 1-year OS rates were $32 \%$ and $48 \%$ for DVD and DD treated patients; these results may well be superior to $23 \%$ survival rate seen in our study, in which none of the VH NT-proBNP patients were treated with daratumumab though our population is a newly diagnosed population, and theirs is a previously treated population. In the Heidelberg study, none of the patients stopped treatment with daratumumab because of toxicity. making it a potentially appealing treatment option for study among patients with VH NT-proBNP.

Although other retrospective studies have evaluated "ultra-high risk" amyloidosis [13-15], focusing on the cardiac stage, we approached the problem from a pragmatic standpoint. Our goal was to define those populations who are typically included and excluded from clinical trials, i.e., using the NT-proBNP threshold of $8500 \mathrm{ng} / \mathrm{L}$ as defined by Wechalekar et al. [4]. We found that $18 \%$ of newly diagnosed AL amyloidosis patients seen at the Mayo Clinic had an NT-proBNP $\geq 8500 \mathrm{ng} / \mathrm{L}$ and consistent with other reports had a poor OS with $74 \%$ dead in the first year. Importantly, survival by stage is considerably better once the VH NT-proBNP patients are removed. This has important implications for trial design using this important variable.

This study is unique in that it compares a cohort of patients with VH NT-proBNP to patients without VH NTproBNP levels, and this comparison yielded several interesting observations, but it does have several limitations. It is a retrospective single-center study performed at a tertiary 
center, and the induction regimens were not uniform. Despite these limitations, the data are important since they can serve as a potential benchmark for expected outcomes both among the sickest AL amyloidosis patients and among patient groups from which the sickest patients have been excluded. Patients with VH NT-proBNP should be considered for specially designed trials, in pursuit of personalized treatment.

Funding This project was supported in part by Grant Number P30 CA015083 and CA186781 from the National Cancer Institute and by the Predolin Foundation and the JABBS Foundation.

\section{Compliance with ethical standards}

Conflict of interest The authors declare no competing interests.

Publisher's note Springer Nature remains neutral with regard to jurisdictional claims in published maps and institutional affiliations.

Open Access This article is licensed under a Creative Commons Attribution 4.0 International License, which permits use, sharing, adaptation, distribution and reproduction in any medium or format, as long as you give appropriate credit to the original author(s) and the source, provide a link to the Creative Commons license, and indicate if changes were made. The images or other third party material in this article are included in the article's Creative Commons license, unless indicated otherwise in a credit line to the material. If material is not included in the article's Creative Commons license and your intended use is not permitted by statutory regulation or exceeds the permitted use, you will need to obtain permission directly from the copyright holder. To view a copy of this license, visit http://creativecommons. org/licenses/by/4.0/.

\section{References}

1. Gertz MA. Immunoglobulin light chain amyloidosis: 2020 update on diagnosis, prognosis, and treatment. Am $\mathbf{J}$ Hematol. 2020;95:848-60.

2. Kumar S, Dispenzieri A, Lacy MQ, Hayman SR, Buadi FK, Colby $\mathrm{C}$, et al. Revised prognostic staging system for light chain amyloidosis incorporating cardiac biomarkers and serum free light chain measurements. J Clin Oncol. 2012;30:989-95.

3. Dispenzieri A, Gertz MA, Kyle RA, Lacy MQ, Burritt MF, Therneau TM, et al. Serum cardiac troponins and N-terminal probrain natriuretic peptide: a staging system for primary systemic amyloidosis. J Clin Oncol. 2004;22:3751-7.
4. Wechalekar AD, Schonland SO, Kastritis E, Gillmore JD, Dimopoulos MA, Lane T, et al. A European collaborative study of treatment outcomes in 346 patients with cardiac stage III AL amyloidosis. Blood. 2013;121:3420-7.

5. Muchtar E, Gertz MA, Kumar SK, Lacy MQ, Dingli D, Buadi FK, et al. Improved outcomes for newly diagnosed AL amyloidosis between 2000 and 2014: cracking the glass ceiling of early death. Blood. 2017;129:2111-9.

6. Schulman A, Connors LH, Weinberg J, Mendelson LM, Joshi T, Shelton AC, et al. Patient outcomes in light chain (AL) amyloidosis: The clock is ticking from symptoms to diagnosis. Eur $\mathbf{J}$ Haematol. 2020;105:495-501.

7. Palladini G, Kastritis E, Maurer MS, Zonder J, Minnema MC, Wechalekar AD, et al. Daratumumab plus CyBorD for patients with newly diagnosed AL amyloidosis: safety run-in results of ANDROMEDA. Blood. 2020;136:71-80.

8. Kastritis E, Leleu X, Arnulf B, Zamagni E, Cibeira MT, Kwok F, et al. Bortezomib, Melphalan, and Dexamethasone for LightChain Amyloidosis. J Clin Oncol. 2020:Jco2001285.

9. Gertz MA, Comenzo R, Falk RH, Fernand JP, Hazenberg BP, Hawkins PN, et al. Definition of organ involvement and treatment response in immunoglobulin light chain amyloidosis (AL): a consensus opinion from the 10th International Symposium on Amyloid and Amyloidosis, Tours, France, 18-22 April 2004. Am J Hematol. 2005:79:319-28.

10. Muchtar E, Kumar SK, Gertz MA, Grogan M, AbouEzzeddine OF, Jaffe AS, et al. Staging systems use for risk stratification of systemic amyloidosis in the era of high-sensitivity troponin $\mathrm{T}$ assay. Blood. 2019;133:763-6.

11. Lousada I, Comenzo RL, Landau H, Guthrie S, Merlini G. Light chain amyloidosis: patient experience survey from the amyloidosis research consortium. Adv Ther. 2015;32:920-8.

12. Kimmich CR, Terzer T, Benner A, Dittrich $\mathrm{T}$, Veelken $\mathrm{K}$, Carpinteiro A, et al. Daratumumab for systemic AL amyloidosis: prognostic factors and adverse outcome with nephrotic-range albuminuria. Blood. 2020;135:1517-30.

13. Shen KN, Zhang CL, Tian Z, Feng J, Wang YN, Sun J, et al. Bortezomib-based chemotherapy reduces early mortality and improves outcomes in patients with ultra-high-risk light-chain amyloidosis: a retrospective case control study. Amyloid. 2019;26:66-73.

14. Manwani R, Foard D, Mahmood S, Sachchithanantham S, Lane T, Quarta C, et al. Rapid hematologic responses improve outcomes in patients with very advanced (stage IIIb) cardiac immunoglobulin light chain amyloidosis. Haematologica. 2018;103:e165-8.

15. Jaccard A, Comenzo R, Hari P, Hawkin P, Roussel M, Morel P, et al. Efficacy of bortezomib, cyclophosphamide and dexamethasone in treatment-naïve patients with high-risk cardiac AL amyloidosis (Mayo Clinic stage III). Haematologica. 2014;99:1479-85. 